\author{
Sinkovics, Rudolf R./Klausegger, Claudia/Floh, Arne
}

\title{
Messung interner Dienstleistungsqualität bei der Ableistung unfreiwilliger Dienstleis- tungen am Beispiel des Osterreichischen Roten Kreuzes
}

\begin{abstract}
Der vorliegende Beitrag beschäftigt sich mit der Messung unfreiwilliger Dienstleistungen gezeigt am Fallbeispiel des Österreichsichen Roten Kreuzes/ Landesverband Niederösterreich. Neben einer praktischen Hilfestellung für die Non-P rofit Organisation (Akquisition von neuen Mitarbeitern durch Überleitung von „unfreiwilligen“ Zivildienern in den Freiwilligendienst nach Absolvierung des Zivildienstens) werden mit der Betrachtung der Unfreiwilligkeit und der internen Dienstleistungsqualität zwei neue Einflussfaktoren geliefert. Für die SERVQUAL-Forschung bedeutet dies einen neuen Anwendungsfall und somit die Erweiterung des Gültigkeits bereiches des populären Ansatzes.
\end{abstract}

Key Words: Qualitätsmessung, Mitarbeiterzufriedenheit, Akquisition und Motivation ehrenamtlicher Mitarbeiter

Dr. Rudolf R. Sinkovics, Univ.Ass. an der Abteilung Internationales Marketing und Management, Wirtschaftsuniversität Wien. E-mail: Rudolf.Sinkovics@wu-wien.ac.at

Dr. Claudia Klausegger, Univ.Ass. an der Abteilung Marketing, Wirtschaftsuniversität Wien.

E-mail: Claudia.Klausegger@wu-wien.ac.at

Mag. Arne Floh, Univ. Ass. an der Abteilung Marketing, Wirtschaftsuniversität Wien.

Email: Arne.Floh@wu-wien.ac.at

\section{Problemstellung und Ziel- setzung}

Das Österreichische Rote Kreuz (ÖRK) gehört seit seiner Gründung 1880 zu einem zentralen Faktor im österreichischen Rettungs- und Krankentransportwesen. In den insgesamt 9 Landesverbänden, 141 Bezirksstellen und 978 Ortsstellen waren $1999 \mathrm{ca}$. 3000 Hauptberufliche, mehr als 38.000 freiwillige Mitarbeiter und 2153 Zivildienstleistende tätig (vgl. http://www.redcross.or.at/rc wer/wer org.htm, Leistungsbericht 1999). Die Zahlen zeigen deutlich, dass das Rote Kreuz den wesentlichen Teil seiner benötigten Arbeitsleistung durch den Einsatz freiwilliger und geringfügig bezahlter Mitarbeiter bezieht. Die von den Freiwilligen geleisteten Arbeit betrug 1999 insgesamt 11,6 Millionen Einsatzstunden. Eine der Hauptaufgaben des Organisations- und Verwaltungsapparates des ÖRK besteht daher neben der Koordination der Einsätze (1999 wurden 2.171.412 Patienten im Rettungs- und Krankentransportdienst betreut) v.a. auch in der Bereitstellung von ausreichend qualifiziertem Personal.

Der vorliegende Beitrag beschäftigt sich in methodischer Hinsicht mit der Messung der internen Dienstleistungsqualität bei der Ableistung des „unfreiwilligen" Zivildienstes auf der Grundlage des auf den Anwendungskontext hin angepaßten SERVQUAL-Ansatzes. Der Focus des Forschungsinteresses liegt dabei auf der Messung von Erwartungen und Wünschen in einem „unfreiwilligen“ Dienstleistungskontext. Wenngleich bereits viele Ansätze und empirische Untersuchungen zur O perationalisierung der Dienstleistungsqualität existieren, stellt der konkrete Anwendungsfall einen innovativen Zutritt dar, weil es nicht um „externe“ Kundenbeziehungen geht, sondern die Beziehung zu „internen“ Kunden im Zentrum des Erkenntnisinteresses steht. 
In praktischer Hinsicht wird mit dieser Arbeit das Ziel verfolgt, Ansätze für eine Steigerung der „Übertrittsquote" (d.i. der Eintritt in eine auf Freiwilligkeit basierende Mitarbeit beim Roten Kreuz nach Beendigung des verpflichtenden Zivildienstes) zu finden. Dies stellt ein konkretes Managementproblem für den Landesverband Niederösterreich dar, der einen Rückgang der jugendlichen Freiwilligen verzeichnet und den organisatorischen Kontext für die gegenständliche Untersuchung bildet. Der Landesverband Niederösterreich sucht nach einer Strategie, die den weiteren Rückgang der für die Aufgabenerfüllung erforderlichen Zahl an freiwilligen M itarbeitern verhindert. Zivildiener stellen für die Akquisition freiwilliger Mitarbeiter eine Kernzielgruppe dar, weil sie neben ihrer zeitlich begrenzten Unterstützung auch ein Potential zukünftiger, ehrenamtlicher Mitglieder darstellen. Das Zivildienermodell in Österreich unterstützt das österreichische Rote Kreuz jährlich mit mehr als 2000 Zivildienern, die zumindest 12 Monate die Arbeit der Organisation unterstützen - dem Landesverband Niederösterreich wurden 1998 davon insgesamt 506 Zivildienstleistende zugeteilt.

Die Annäherung an den dargestellten Problemkreis erfolgt über die Messung der Arbeitszufriedenheit der Zivildienstleistenden. Durch die systematische Erfassung deren Erwartungen, Wünsche und Erfahrungen im Rahmen ihrer Tätigkeit, sollen bestehende Defizite aufgezeigt werden, die als Ausgangspunkt für längerfristige Veränderungsprozesse genutzt werden können. Ergänzt wird dieser Ansatz um organisationstheoretische Komponenten, die den Hintergrund zum Verständnis der M otivstruktur freiwilliger Arbeit in Non-Profit Organisationen liefern.

\section{Konzeptioneller Rahmen der Untersuchung}

\subsection{Begriffsklärung}

Unter ehrenamtlicher Arbeit wird eine Arbeitsleistung verstanden, der kein monetärer Geldfluss gegenübersteht, die also nicht mit Geld bezahlt wird (Pearce, 1993, S. 8). Zu den typischen Abgrenzungsund Beschreibungsmerkmalen zählen die Freiwilligkeit, Ehrenamtlichkeit, Unentgeltlichkeit, „zum Wohle Anderer", in organisierter Form, Nebenberuflichkeit, und "Laienarbeit" (vgl. Strümpel/Pleschberger/ Riedel, 1999, S. 7; Höflacher, 1999, S. 52ff.). Auf die Tätigkeit der Zivildienstes sind diese Merkmale nur zum Teil zutreffend, weil im Falle des Zivildienstes ein ganz spezifisches Beschäftigungsverhältnis vorliegt. Zivildienstleistende sind über einen Zeitraum von zwölf Monaten aufgrund einer gesetzlichen Zwangsverpflichtung in sozialen NPOs tätig (vgl. v. Eckardstein, 1997, S. 259). Trotz dieser gesetzlichen Verpflichtung, gilt es allerdings zu klären, welche Gründe für die Wahl des Zivildienstes beim Roten Kreuz sprechen. Trotz des inhärenten Zwangscharakters der Zivildiensttätigkeit bieten die Motivationsfaktoren ehrenamtlicher Mitarbeiter aus der Organisationspsychologie eine geeignete konzeptionelle Basis.

\subsection{Organisationspsyc hologisc he Theorieansätze zur Motivati- onsstruktur ehrenamtlic her Mitarbeiter}

Grundsätzlich wird in der Literatur zur Organisationspsychologie zwischen extrinsischer und intrinsischer Motivation unterschieden. Intrinsisch motiviertes Verhalten liegt dann vor, wenn die ausführende Person B efriedigung aus der Tätigkeit selbst erlangt, Rosenstiel (2000) führt als intrinsische Arbeitsmotive das Bedürfnis nach Tätigkeit, das Kontaktbedürfnis, Leistungsmotivation, Machtbedürfnis und das Bedürfnis nach Sinngebung und Selbstverwirklichung an (vgl. v. Rosenstiel, 2000, S. 359). Extrinsische Motivation liegt dann vor, wenn die Tätigkeit weniger aus freien Stücken als aufgrund externaler Handlungsfolgen gesetzt wird. Zu den extrinsischen Arbeitsmotiven zählen das Bedürfnis nach Geld, Konsumbedürfnisse, Sicherheitsbedürfnisse, Geltungsstreben und Kontaktbedürfnis ( $v$. Rosenstiel, 2000, S. 359). Das Ziel der Motivationstheorien ist es, das "Warum" des menschlichen Handelns zu erklären. Dazu zählen sowohl Inhaltstheorien vgl. Theorie der Bedürfnishierarchie (Maslow), ERG Theorie (Alderfer), Zwei-Faktoren-Theorie (Herzberg); Prozesstheorien Z.B. Erwartungs-Valenz-Theorie (Atkinson), VIE-Theorie (Vroom), Equity-Theorie (Adams) als auch sog. gemischte Ansätze wie die Leistungsmotivationstheorie (McClelland/Atkinson) und die J ob Characteristics Theory (Hackman/Oldham). Da vorangegangene empirische Untersuchungen betreffend der Motivation zur Mitarbeit beim Roten Kreuz gezeigt haben, dass es dabei vor allem um die Begriffe wie Sinnhaftigkeit und Verantwortung geht, ist v.a. die J ob Characteristics Theory als Theorieansatz von Interesse (vgl. auch Schildorfer 2000). Die J ob Characteristics Theory wurde 1974 von den beiden Arbeitspsychologen Hackman und 
Oldham entwickelt. Dem Modell liegt ein humanistisches Menschenbild zugrunde, das durch eine Tendenz zur Selbstverwirklichung gekennzeichnet ist. Grundlage der J CT ist das Modell der Bedürfnishierachie und die Erwartungsvalenz-Theorie (vgl. Mayrhofer, 1996, S. 249). Die Kernfragestellung lautet: Wie kann man Arbeit gestalten, damit sie einerseits effektiv ist und andererseits für den Arbeitnehmer lohnend und zufriedenstellend empfunden wird (vgl.
Hackman, Oldham, 1980, S. 71ff.). Als Voraussetzung der intrinsische Motivation führen Hackman/ Oldham folgende drei Punkte an: die Ergebnisse der Arbeit kennen, die Verantwortung prüfen und Arbeit als sinnvoll erachten.

Die Sinnhaftigkeit der Arbeit wird vor allem durch die Aufgabenvielfalt, die Aufgabenidentität und die Aufgabenbedeutung beeinflusst.

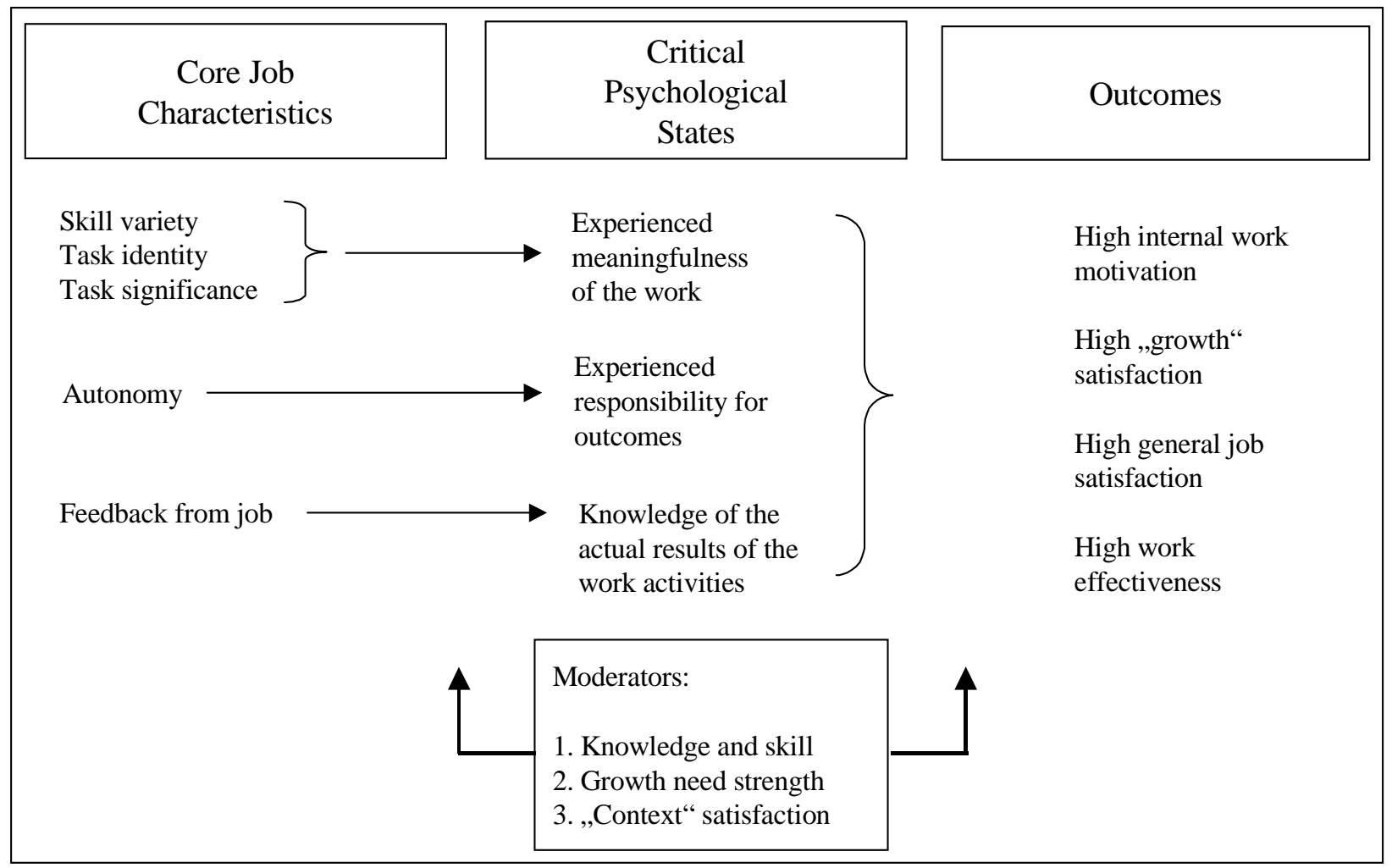

Abbildung 1: Die J ob Characteristics Theory (Hackman/Oldham, 1980)

Folgt man diesem Theorieansatz, müsste es eines der Kernziele des ÖRK sein, die intrinsischen Motive v.a. für ihre freiwilligen Mitarbeiter zu sichern und die Arbeitsplatzkomponenten entsprechend zu gestalten. Da aber nicht alle Menschen auf die gleiche Weise auf die zentralen Tätigkeitsbereiche, die kritischen psychischen Zustände und die Ergebnisse aus der Arbeit reagieren, müssen für die Bestimmung des Motivationspotentials auch die persönlichen Eigenschaften von Personen berücksichtigt werden. Dazu zählen neben dem Wissen, den Fähigkeiten und Fertigkeiten, die Stärke der Wachstumsbedürfnisse und die Zufriedenheit mit dem Arbeitskontext. Die Zufriedenheit mit dem Arbeitskontext beeinflusst den
Willen und die Fähigkeit, die persönliche Leistung zu entfalten. Personen, die eher zufrieden sind, reagieren positiver auf herausfordernde Arbeit als unzufriedene.

\subsection{Zusammenhang zwischen Erwartungen, Zufriedenheit und Verhalten}

Der vorliegende Beitrag geht von dem in der Literatur postulierten Zusammenhang zwischen Arbeitszufriedenheit und persönlicher Leistungsbereitschaft 
und -fähigkeit aus und erweitert diesen durch die in der empirischen organisationspsychologischen Forschung nahezu unbestrittene These, dass eine Organisation durch die präventive Erfassung von Erwartungen potentieller bzw. zukünftiger Mitarbeiter Marktvorteile erreichen kann. (vgl. Schwaab, 1993) Während der Qualitätsmanagementgedanke und die Sicherung von Qualitätsaspekten externer Kunden bereits zum Alltagsverständnis moderner Unternehmensphilosophien zählt, müssen innerhalb der Organisation häufig Defizite festgestellt werden, obwohl es konzeptionell nahe liegt, zumindest die Grundprinzipien des externen Qualitätsmarketing auch auf innerbetriebliche Beziehungen anzuwenden, in der Form, dass die Grundlage für eine hohe Dienstleistungsqualität in der Erfüllung der Erwartungen liegt und deren Nichterfüllung Konsequenzen für den betroffenen „Kunden“ hat. (vgl. Roßbach-Ebden/Gaalken/Pauli, 1995, S. 511f.).

Der vorliegenden Untersuchung liegt daher die Annahme zugrunde, dass Erwartungen und ihre Erfüllung bzw. Nichterfüllung einen wesentlichen Einfluss auf das Handeln der Zivildiener haben. Natürlich muss der Zusammenhang zwischen den Faktoren Erwartungen, Zufriedenheit und Handeln auf unterschiedliche Bedingungsfaktoren Rücksicht nehmen. Dennoch zeigen aber die wirtschaftspsychologische Studien, die dem komplexen Beziehungsgefüge gerecht werden, einen Zusammenhang zumindest zwischen dem Fluktuationsverhalten und den nicht erfüllten Erwartungen von M itarbeitern. Vor allem bei Neueinsteigern in Organisationen lässt sich untersuchen, ob und welche Erwartungen an die Organisation gestellt werden und welche Auswirkungen das Erleben von Diskrepanzen bei der Nichterfüllung von Erwartungen nach sich ziehen. Nach Steers/M owday (1981) hat jeder Mitarbeiter hinsichtlich einer neuen Arbeitssituation bestimmte Erwartungssets, die sich auf unterschiedliche Facetten wie die Bezahlung, das Betriebsklima oder die Beförderungsmöglichkeiten beziehen. In seinen theoretischen Annahmen zum Fluktuationsverhalten geht Steers davon aus, dass in Abhängigkeit der Erwartungen und dem Grad der im Laufe der Organisationsmitgliedschaft erfüllten Erwartungen der Mitarbeiter mit positivem oder negativen Einstellungen gegenüber der Organisation reagiert wird. J e mehr negative Einstellungen gegenüber der Arbeit und der Organisation existieren, desto größer wird die Absicht, die Organisation zu verlassen. Lee/Mowday (1987) konnten in ihrer Studie zum Fluktuationsverhalten von Bankangestellten zeigen, dass die Erwartungserfüllung, die subjektive Wertigkeit der Arbeits- facetten, die wahrgenommenen Organisationscharakteristika, die Erfahrungen innerhalb der Organisation und die Arbeitsleistung zusammen auf verschiede Einstellungsgrößen wirken (Arbeitszufriedenheit, Organisationscommitment, J ob Involvement). (vgl. Roßbach-Ebden/Gaalken/Pauli, S. 514f.) Zusammengefasst zeigen nahezu alle Fluktuationsstudien, dass unter Berücksichtigung eines großen Spektrums unterschiedlicher Einflussgrößen, die Nichterfüllung von Erwartungen Auswirkungen auf das menschliche Handeln hat.

\subsection{Servqual-Ansatz zur Messung der internen Dienstleistungs- qualität}

Als M ethoden zur Messung der internen Dienstleistungsqualität stehen zahlreiche Verfahren aus dem externen Dienstleistungsbereich zur Verfügung z.B. Frequenz-Relevanz-Analyse von Problemen, Sequentielle Ereignismethode, Critical-Incident-Technik und interne Kunden-/M itarbeiterbefragungen. Im vorliegenden Projekt wurde auf den SERVQUALAnsatz (Parasuraman/Zeithaml/Berrys, 1985, 1988) als ein einstellungsorientiertes multiattributives Qualitätsmessverfahren abgestellt. Dienstleistungsqualität wird dabei als globales einstellungsorientiertes Konstrukt interpretiert, das aus dem Vergleich zwischen erwarteter und erlebter Leistung resultiert (Hentschel, 1990a, S. 232). Zur Messung der wahrgenommenen Dienstleistungsqualität mit Hilfe des SERVQUAL-Ansatzes wird ein standardisierter Fragebogen entworfen, in dem 22 Items fünf Dimensionen der Qualität repräsentieren: Annehmlichkeit des tangiblen Umfeldes, Zuverlässigkeit, Reaktionsfähigkeit, Leistungskompetenz, Einfühlungsvermögen. Zu jedem Item werden zwei Aussagen in Form einer Doppelskala formuliert. Die Aussage „so sollte es sein" ermittelt die Erwartungen hinsichtlich der Dienstleistungsqualität, die Aussage "so ist es“ fragt nach der erlebten Qualität des Leistungsprozesses. Auf einer 7-teiligen Skala werden die Befragten gebeten, ihr Urteil von „stimme völlig zu“ (7) bis "lehne entschieden $a b$ “ (1) abzugeben. Die sich ergebende Differenz zwischen beiden Aussagen ergibt dann einen Wert pro Item zwischen -6 und +6. J e größer der Wert, umso höher wird die wahrgenommene Dienstleistungsqualität eingeschätzt.

Um ein globales Qualitätsurteil zu erhalten, wird zunächst der Durchschnitt der zu einer Dimension gehörenden Items berechnet und anschließend der 
Mittelwert sämtlicher Dimensionen ermittelt. Trotz der empirischen Fundierung des Modells und seiner grundsätzlichen Eignung als branchenunabhängiges Modell zur Messung der Dienstleistungsqualität, gibt es in der Literatur mehrfach kritische Äußerungen betreffend der begrifflichen und theoretischen Grundlagen sowie der methodischen Aspekte des Modells (vgl. u.a. Carman, 1990; Hentschel, 1990a, 1990b).

Hauptkritikpunkte sind dabei, die hohen Ansprüche an die Urteilsfähigkeit aufgrund der Zerlegung der gemachten Erfahrungen hinsichtlich der verschiedenen Dimensionen in eine Erwartungs- und Wahrnehmungskomponente und das Problem der Erwartungsinflation. Ein weiteres Problemfeld liegt in der Differenzbildung des Modells. Darüber hinausgehend ist eine eindeutige Interpretation in bezug auf die Erwartungen nicht immer gewährleistet, da die Aussage „so sollte es sein“ auf unterschiedliche Weise interpretiert werden kann (Teas, 1993, S. 37f.):

- als reine Annahme über das Niveau der Dienstleistungsqualität /(„Forecasted Performance“)

- als gewünschtes Niveau der Dienstleistungsqualität („Deserved Performance“)

- als angemessenes Niveau der Dienstleistungsqualität („Equitable Performance“)

- als Mindestniveau der Dienstleistungsqualität (,Minimum Performance“)

- als Idealniveau der Dienstleistungsqualität („Ideal Performance") oder

- als Ausdruck der Wichtigkeit dieser Dimension der Dienstleistungsqualität für den Zivildiener („Service Attribute Importance“).

Die an späterer Stelle präsentierten Messergebnisse müssen daher vor dem Hintergrund unterschiedlicher Interpretationsmöglichkeiten des Erwartungsbegriffes interpretiert werden. Trotz dieser dargestellten Problemfelder in bezug auf die grundsätz- liche Validität des Modellansatzes hat sich der SERVQUAL-Ansatz zur Messung der Dienstleistungsqualität etabliert und wird aus den bereits genannten Gründen für die vorliegende empirische Studie herangezogen. Da für das vorliegende Untersuchungsobjekt auf keine bereits getestete Messbatterie abgestellt werden konnte, musste eine neues modifiziertes Messinstrument entwickelt werden, das den Anforderungen und Besonderheiten der Erhebungssituation bzw. des Zivildienstes beim ÖJ RK gerecht wird.

\section{Darstellung des Mess- instruments und des Erhe- bungsdesigns}

Das im vorliegenden Anwendungsfall eingesetzte Erhebungsinstrument zur Messung der Zufriedenheit (Erwartungen und Erfahrungen) der Zivildiener mit ihrer Tätigkeit beim Roten Kreuz Niederösterreich wurde als zweistufige anonyme Längsschittsuntersuchung konzipiert. Die Zuordnung der Fragebögen der beiden Wellen erfolgte über einen anonymisierten Identifikationscode.

\subsection{Zeit und Ort der Erhebung}

Die erste Erhebung wurde als Vollerhebung am 2.2.1997 in der Kommandozentrale des Landesverbandes Niederösterreich in Tulln durchgeführt. Die Zivildiener wurden bei Dienstantritt durch ein Informationsblatt informiert und gebeten den Fragebogen auszufüllen.

Die zweite Erhebung erfolgte im J uni 1997 durch postalische Zusendung der schriftlichen Fragebögen an die jeweilige Dienststelle des ÖRK/LV NÖ.

\begin{tabular}{|c|c|c|}
\hline Erhebungswelle & Erhebungszeitraum & Stichprobenumfang \\
\hline 1 & $\begin{array}{c}2.2 .1997 \\
\text { (Einrückungstag der Zivildiener) }\end{array}$ & $\begin{array}{c}\mathrm{n}=179 \text { verwertbare Fragebögen } \\
\text { (mit Identifikationscode) }\end{array}$ \\
\hline 2 & J uni 1997 & $\begin{array}{c}\mathrm{n}=84 \text { verwertbare Fragebögen } \\
\text { (mit Identifikationscode) }\end{array}$ \\
\hline
\end{tabular}

Tab. 1: Daten der Erhebungswellen 


\subsection{Aufbau und Beschreibung des Fragebogens der ersten Er- hebungswelle (Erwartungen der Zivildiener)}

Der erste Teil des Fragebogens der ersten Erhebungswelle umfasst 15 teilweise offen gestellte Fragen betreffend

- der Motive und Gründe für die Zivildienstentscheidung generell und der Wunsch nach Ableistung beim Roten Kreuz

- des Informationsstandes und den bisherigen Erfahrungen mit dem Roten Kreuz

- der Wahrscheinlichkeit nach Absolvierung des Zivildienstes als freiwilliger Mitarbeiter beim Roten Kreuz mitzuarbeiten und

- den Erwartungen hinsichtlich der Ableistung des Zivildienstes beim Roten Kreuz.

Der zweite Teil basiert auf dem SERVQUAL-Ansatz und enthält 50 Fragen zu insgesamt neun Kategorien. Die ursprüngliche SERVQUAL-Version von Parasuraman/Zeithaml/Berry, bestehend aus den fünf Kategorien (tangibles Umfeld allgemein, Verläßlichkeit, Reagibilität, Leistungskompetenz allgemein und Einfühlungsvermögen, wurde leicht modifiziert und um vier weitere NPO relevante Kategorien (tangibles Umfeld speziell, Leistungskompetenz speziell, psychische Ebene und Aufgabenbereich) erweitert.

Dementsprechend waren sowohl eine Reihe von allgemeinen Aussagen über das Österreichische Rotes Kreuz als auch Aussagen, welche speziell das Arbeitsumfeld der Zivildiener betreffen sollten, eingeschlossen.

Ergänzt wurde der Fragebogen durch 35 Fragen zu den folgenden vier Konstrukten:

- Meinungsführerschaft bzw. Opinionleadership (OLS) (7 items, 5-stufig skaliert)

- Materialismus (6 items, 5-stufige Ratingskala)

- Soziale Erwünschtheit (7 items, 5-stufige Ratingskala) und

- Involvement (semantisches Differential, 15 items, 7-stufig skaliert)

Die folgenden deskriptiven Informationen bildeten den Abschluß des Fragebogens: Ausbildung, Beruf, Familienstand, Alter, rel. Bekenntnis, pol. Bezirk, Vereinszugehörigkeit.

\subsection{Aufbau und Beschreibung des Fragebogens der zweiten Er- hebungswelle (Erfahrungen der Zivildiener)}

Der Fragebogen der zweiten Welle enthält fünf Fragen betreffend positiver und negativer Ereignisse während des Zivildienstes (Erfahrungen) und hinsichtlich der Übertrittswahrscheinlich als freiwilliger Helfer. Der zweite Fragenblock ermittelt anhand von fünfzig Items die Erfahrungen der Auskunftspersonen während des Zivildienstes basierend auf dem SERVQUAL-Ansatz. Der dritte Teil testet die Veränderungen über die Zeit hinsichtlich der beiden Konstrukte Materialismus und Involvement ab; zusätzlich wurde eine Item-Batterie zur Einstufung der Motivation der Zivildiener aufgenommen (8 Items, 7-stufige Ratingskala).

\section{Ausgewählte Untersuchungs- ergebnisse}

Die Darstellung der Ergebnisse erfolgt analog zum beschriebenen Forschungs- und Erhebungsdesign. Nach einer Darstellung ausgewählter Ergebnisse der beiden Erhebungszeiträume $t_{1}$ (Motive für die Wahl des Zivildienstes, Informationsquellen, Ängste und Befürchtungen) und $t_{2}$ (positive und negative Erlebnisse, Zufriedenheit,Üb ertrittswahrscheinlichkeit) im Rahmen der deskriptiven Statistik, erfolgt die Prüfung der beiden Basishypothesen des SERVQUALAnsatzes, sowie einiger weiterer demografischer und psychografischer Zusammenhangshypothesen.

\subsection{Deskriptive Ergebnisse der ersten Welle $\left(t_{1}\right)$}

\subsubsection{Motive für die Wahl des Zivildienstes}

Im Rahmen der ersten Erhebungswelle wurde versucht, die möglichen Motive bzw. B eweggründe für die Entscheidung der Auskunftspersonen zum Zivildienst zu gehen - als Alternative zur Absolvierung des Präsenzdienstes beim Österreichischen Bundesheer - herauszufiltern. Aufgrund der Antworten der Befragten wurden mittels einer Multiple Response Analysis acht Kategorien, die für die Wahl des Zivildienstes entscheidend sind, ermittelt (vgl. Tabelle 2) 


\begin{tabular}{|l|c|c|c|}
\hline \multicolumn{1}{|c|}{ Gründe } & $\begin{array}{c}\text { Anzahl der } \\
\text { Nennungen } \\
\text { (absolut) }\end{array}$ & $\begin{array}{c}\text { Anzahl der } \\
\text { Nennungen } \\
\text { (in \%) }\end{array}$ & $\begin{array}{c}\text { Anzahl der } \\
\text { Befragten } \\
\text { (in \%) }\end{array}$ \\
\hline Abneigung gegen BH, Flucht vor dem BH & 59 & 26,5 & 34,9 \\
\hline Bereits (bzw. Wunsch) Mitarbeiter des ÖRK & 5 & 2,2 & 3,0 \\
\hline Interessante, sinnvolle Tätigkeit(en) & 48 & 21,5 & 28,4 \\
\hline Interesse, Spaß am Sozialdienst & 41 & 18,4 & 24,3 \\
\hline Gewissensgründe, moralische Gründe & 59 & 26,5 & 34,9 \\
\hline Flexiblere Diensteinteilung & 5 & 2,2 & 3,0 \\
\hline Bessere Bezahlung & 2 & 0,9 & 1,2 \\
\hline Nähe zur Dienststelle & 4 & 1,8 & 2,4 \\
\hline Summe & 223 & 100,0 & 132,0 \\
\hline
\end{tabular}

Tab. 2: Motive für die Wahl des Zivildienstes

Von den acht Kategorien wurden die Kriterien „Abneigung gegenüber dem Bundesheer" bzw. „Gewissensgründe, moralische Gründe " am häufigsten (absolut) genannt. Dieses Ergbenis entspricht jeweils ca. einem Drittel aller befragten Zivildiener. Knapp dahinter folgen die Motive „Interesse, S paß am Sozialdienst" mit 28,4\% und "Interesse, Spaß am Sozialdienst" mit 24,3 \% (48 bzw. 41 Nennungen). Nahezu unbedeutend im Vergleich dazu sind die restlichen Kategorien. So gaben nur etwa $3 \%$ aller Zivildiener "flexible Diensteinteilung“ als Beweggrund an.

\subsubsection{Informationsquellen der Zivildiener}

Einen Schwerpunkt der vorliegenden Studie im Hinblick auf die Kommunikationstätigkeit des Roten Kreuzes bildete die Frage nach dem Informationsstand und Meinungsbildungsprozess der Auskunftspersonen. Um dieser Fragestellung gerecht zu werden, wurde neben dem Konstrukt des „Opinion-Leader-Ship" auch das Informationsverhalten der Zivildiener vor Antritt des Zivildienstes erhoben.

\begin{tabular}{|l|r|r|r|}
\hline $\begin{array}{c}\text { Genutzte Informationsquellen vor Antritt } \\
\text { des Zivildienstes }\end{array}$ & $\begin{array}{c}\text { Anzahl der } \\
\text { Nennungen } \\
\text { (absolut) }\end{array}$ & $\begin{array}{c}\text { Anzahl der } \\
\text { Nennungen } \\
\text { (in \%) }\end{array}$ & $\begin{array}{c}\text { Anzahl der } \\
\text { Befragten } \\
\text { (in \%) }\end{array}$ \\
\hline Freunde, Bekannte, Verwandte & 131 & 39,6 & 73,2 \\
\hline Medien (Fernsehen, Zeitungen etc.) & 21 & 6,3 & 11,7 \\
\hline Mitarbeiterlnnen des RK & 81 & 24,5 & 45,3 \\
\hline Informationsbroschüre & 29 & 8,8 & 16,2 \\
\hline Bezirksdienststelle & 27 & 8,2 & 15,1 \\
\hline Berufliche Tätigkeit & 4 & 1,2 & 2,2 \\
\hline Eigene Tätigkeit beim RK & 26 & 7,9 & 14,5 \\
\hline Keine Information & 8 & 2,4 & 4,5 \\
\hline Zivildiener & 1 & 0,3 & 0,6 \\
\hline Erste-Hilfe-Kurs & 1 & 0,3 & 0,6 \\
\hline Allgemeinwissen & 2 & 0,6 & 1,1 \\
\hline (0 missing cases, 179 valid cases) & 331 & 100,0 & 184,9 \\
\hline
\end{tabular}

Tab 3: Informationsquellen der Zivildiener

Von den 11 zur Auswahl gestellten Informationsquellen wurden „Freunde, Bekannte, Verwandte“ (131 Nennungen) und M itarbeiter des Roten Kreuzes (81 Nennungen) mit Abstand am häufigsten genannt. Bezogen auf die Zahl der B efragten bedeutet dies, dass sich $73 \%$ der Befragten vor dem Antritt des Zivildienstes bei "Freunden, Bekannten und Verwandten“ informiert hatten. „Klassische Werbeträger" spielen hingegen nur eine untergeordnete Rolle. So gab nur ein geringer P rozentsatz aller Befragten an, sich mittels B roschüre $(16,7 \%)$ oder Medien $(11,7 \%)$ informiert zu haben. 


\section{2. Ängste und Befürchtungen}

Im Zuge der Erhebung der Erwartungen wurde versucht, mögliche Ängste bzw. unangenehme, wenig wünschenswerte Erlebnisse und Ereignisse der
Zivildiener in bezug auf den bevorstehenden Zivildienst zu erforschen. Hier standen den Auskunftspersonen insgesamt 100 P unkte zur Verfügung, die sie je nach Bedarf auf zehn vorgegebenen Antwortkategorien frei verteilen konnten.

\begin{tabular}{|l|c|}
\hline \multicolumn{1}{|c|}{ Ängste vor Antritt des Zivildienstes } & Gewichtung der Aussage \\
\hline Schlechtes Klima & 16,32 \\
\hline Unfaire Behandlung & 14,37 \\
\hline zu viele Nachtdienste & 5,00 \\
\hline Unangenehme Arbeit & 5,11 \\
\hline Einsätze bei Verwandten und Bekannten & 7,07 \\
\hline Schwere Unfälle, Katastrophen & 9,78 \\
\hline schuld sein, dass jemand stirbt & 23,15 \\
\hline Schlechte Ausbildung & 10,04 \\
\hline eigene psychische oder physische Verletzung & 8,82 \\
\hline Sonstiges & 0,34 \\
\hline Punkte & 100,00 \\
\hline
\end{tabular}

Tab. 4: Ängste und Befürchtungen der Zivildiener ( $n=179)$

Aus der Tabelle 4 wird klar ersichtlich, dass die größte Befürchtung der Zivildiener ein mögliches Verschulden mit Todesfolgen darstellt. Die Antwortkategorie "schuld sein, dass jemand stirbt" wird mit 23,15 von 100 Punkten eindeutig am stärksten gewichtet. Große Bedeutung wird ebenfalls den Bereichen "schlechtes Arbeitsklima“, "unfaire Behandlung " und "schlechte Ausbildung" mit immerhin $16,32,14,37$ und 10,04 Punkten beigemessen.

Relativ gelassen sehen die Zivildiener der M öglichkeit "zu viele Nachtdienste" und "unangenehme Arbeit" entgegen (5,00 bzw. 5,11 Punkte). Dieses Ergebnis unterstreicht die Wichtigkeit einer umfassenden Aus bildung sowie der sozialen Komponente bei Ableistung des Zivildienstes.

\subsection{Ausgewählte Ergebnisse im Beobachtungszeitraum $t_{2}$}

\subsubsection{Erfahrungen der Zivildiener}

Eine Analyse der Mehrfachantworten zu den bisher gewonnenen Eindrücken und Erfahrungen beim Zivildienst zeigte die besondere Bedeutung des sozialen Umfeldes. In der Kollegenschaft dominiert die Zahl der Referenzinformationen sowohl hinsichtlich positiver $(55 \%)$ als auch negativer Eindrücke $(48 \%)$. Auch bei den Eindrücken zu „Einsätzen“ zeigt sich ein dominant ambivalentes Bild. $30 \%$ der Zivildiener gewannen bei ihren Einsätzen positive Eindrükke, $20 \%$ empfanden diesen Bereich als negativ.

\begin{tabular}{|c|c|c|c|c|c|c|c|}
\hline Eindrücke & Kollegen & Vorgesetzte & Patienten & Einsätze & Dienstzeit & Tätigkeit & Unfälle \\
\hline Positiv & $55 \%$ & $3 \%$ & $43 \%$ & $30 \%$ & $22 \%$ & - & - \\
\hline Negativ & $48 \%$ & $18 \%$ & $10 \%$ & $21 \%$ & $27 \%$ & $32 \%$ & $24 \%$ \\
\hline
\end{tabular}

Tab. 5: Positive/negative Eindrücke während des Zivildienstes $(n=179)$

\subsubsection{Zufriedenheit mit der Zivil- diensttätigkeit}

Im Rahmen der Abschlussfrage der zweiten Erhebungswelle wurde die Gesamtzufriedenheit der Zivildiener mit der bisherigen Arbeitserfahrung (5 Monate) erhoben. Dies erfolgte in Ergänzung zum Zu- friedenheitsscore der sich aus dem SERVQUALAnsatz errechnen ließ.

Die Auskunftspersonen hatten dabei die Möglichkeit, ihre Antworten prozentuell abzustufen, wobei 0 $\%$ „überhaupt nicht zufrieden“ und $100 \%$ „sehr zufrieden" bedeutete. 


\begin{tabular}{|c|c|c|c|c|c|c|c|c|c|c|}
\hline $\begin{array}{c}\text { Zufriedenheit } \\
\text { in \% }\end{array}$ & $0-10$ & $11-20$ & $21-30$ & $31-40$ & $41-50$ & $51-60$ & $61-70$ & $71-80$ & $81-90$ & $91-100$ \\
\hline $\begin{array}{c}\text { Zivildiener } \\
\text { in \% }\end{array}$ & 3,7 & 3,6 & 4,9 & 2,4 & 9,8 & 6,1 & 12,2 & 20,7 & 18,3 & 18,3 \\
\hline
\end{tabular}

Tab. 6: Prozentuelle Zufriedenheit der Zivildiener $(n=84)$

Als Ergebnis vergaben fünf Zivildiener $(6,1 \%$ der Grundgesamtheit) die Höchstnote $100 \%$, lediglich ein Zivildiener (1,2\% der Grundgesamtheit) war mit dem bisherigen Zivildienst überhaupt nicht zufrieden $(0 \%)$. Rund ein Viertel aller Auskunftspersonen $(24,4$ $\%$ ) bewerteten den Zivildienst mittelmäßig zufriedenstellend, der Rest der Zivildiener (rund drei Viertel) war mittelmäßig bis sehr zufrieden. Der Durchschnittswert über alle befragten Zivildiener liegt bei $70,3 \%$.

\subsection{Veränderungen im Beo- bachtungszeitraum $t_{1}$ und $t_{2}$}

\subsubsection{Ergebnisse betreffend der Übertrittswahrscheinlichkeiten}

Der vorliegende B eitrag verfolgte neben einer wissenschaftlichen Aufgabenstellung auch das Ziel, eine praktische Hilfestellung für das Österreichische Rote Kreuz - konkret den Landesverband Niederösterreich - zu liefern.

Um diesem Ziel gerecht zu werden, wurde bei beiden Erhebungswellen die Wahrscheinlichkeit für das Eintreten als freiwilliger Mitarbeiter der Auskunftspersonen nach Absolvierung des Zivildienstes erhoben.

\begin{tabular}{|c|c|c|c|c|c|c|c|c|c|c|}
\hline $\begin{array}{c}\text { Übertritts- } \\
\text { wahrschein- } \\
\text { lichkeit in \% }\end{array}$ & $0-10$ & $11-20$ & $21-30$ & $31-40$ & $41-50$ & $51-60$ & $61-70$ & $71-80$ & $81-90$ & $91-100$ \\
\hline Zeitraum $\mathrm{t}_{1}$ & 18,4 & 5,6 & 6,7 & 4,5 & 25,7 & 3,9 & 5,6 & 9,5 & 5,0 & 15,1 \\
\hline Zeitraum $\mathrm{t}_{2}$ & 20,2 & 3,6 & 3,6 & 3,6 & 16,6 & 1,2 & 6 & 7,1 & 10,7 & 27,4 \\
\hline
\end{tabular}

Tab. 7: Übertrittswahrscheinlichkeiten im Zeitablauf $\left(n_{t 1}=179, n_{t 2}=84\right)$

Obenstehende Tabelle 7 zeigt sehr deutlich, dass nach Absolvierung der ersten fünf Zivildienstmonate eine leichte positive Verschiebung der Übertrittswahrscheinlichkeiten der B efragten festgestellt werden konnte. Beträgt der Durchschnittswert bei Antritt des Zivildienstes noch 51,4\%, so zeigt sich nach fünf M onaten (zum Zeitpunkt der zweiten Erhebung) eine Steigerungsrate um 7,7 \% $\left(\right.$ mean $\left._{\mathrm{t} 2}=59,1 \%\right)$.

\subsection{Hypothesenüberprüfung}

\subsubsection{Entwicklung des Servqual- Erhebungsinstruments im Kontext unfreiwilliger Dienstleistungen}

Für die nachfolgende Messung der Qualität im Kontext unfreiwilliger Dienstleistungen wurde auf den Servqual-Meßansatz von Parasuraman, Zeithaml und Berry zurückgegriffen. Parasurman et al. verwendeten diesen Ansatz zur Operationalisierung der Qualität im Kontext von Finanzdienstleistungen. In einer Reihe von Studien (Llosa et al., 1998; Lam et al., 1997; Orwig et al., 1997; Donnelly et al., 1995; Babakus et al., 1992) wurde der Servqual-Ansatz auf spezifische andere Unternehmens-/Dienstleistungsbereiche (Behörden, Spitäler, Universitäten, etc.) erweitert und angepasst. Für den gegenständlichen Anwendungshintergrund der unfreiwilligen Dienstleistungsverrichtung liegt bislang kein Anwendungsfall vor. Insofern galt es der Empfehlung der Autoren nachzukommen und das Itempool an den gegenständ lichen Untersuchungskontext anzupassen. $\mathrm{Zu}$ diesem Zweck wurde im Rahmen einer qualitativen Item-Entwicklungs-P hase (Churchill, 1979) eine Reihe von kontextspezifischen Items generiert und dem ursprünglichen Satz von Items aus Parasuraman et al. hinzugefügt.

Mit Hilfe der explorativen Faktoranalyse wurde aus dem Set von 50 Items jene herausgefiltert, welche 
am besten geeignet sind, das Konstrukt „unfreiwillige Dienstleistungsqualität" zu messen.

Zunächst wurde die Überprüfung der Eignung des Datensatzes auf faktoranalytische Untersuchungen anhand der Kriterien „Kaiser-Meyer-Olkin Measure of Sampling Adequacy“ und „Bartlett's Test of Sphericity" durchgeführt. Nachdem das Ziel der Variablenreduktion eine $\mathrm{M}$ inimalzahl von Faktoren/Dimensio- nen vorsah, welche den Hauptteil der Varianz erklären sollte, und wir aufgrund explorativer Analysen davon ausgehen konnten, daß nur geringe Irrtumsvarianzen vorlagen (vgl. Hair, 1998), wurde die Entscheidung zugunsten einer PCA („P rincipal Components Analysis“) getroffen. Das Ergebnis der Faktoranalyse (Varimax-Rotation) ist in nachfolgender Tabelle dargestellt. Insgesamt resultierte die PCA in ein reduziertes Set von 22 Items und 4 Dimensionen:

\begin{tabular}{|c|c|c|c|}
\hline $\mathrm{Nr}$. & ITEM & $\begin{array}{l}\text { LADUNG } \\
t_{1}\end{array}$ & $\begin{array}{l}\text { LADUNG } \\
\mathrm{t}_{2}\end{array}$ \\
\hline \multicolumn{4}{|c|}{ Dimension Ausbildung } \\
\hline V1 & $\begin{array}{l}\text { Das Leitbild des ÖRK sollte jedem Zivildiener während der Grundausbildung } \\
\text { vermittelt werden. }\end{array}$ & 0,576 & 0,504 \\
\hline V2 & $\begin{array}{l}\text { Die Ausbildung der Zivildiener während des Grundlehrganges sollte fachlich } \\
\text { umfassend sein. }\end{array}$ & 0,722 & 0,608 \\
\hline V3 & $\begin{array}{l}\text { Die Ausbildung der Zivildiener während des Grundlehrganges sollte eine gute } \\
\text { Vorbereitung auf die spätere Tätigkeit sein. }\end{array}$ & 0,595 & 0,774 \\
\hline V4 & $\begin{array}{l}\text { Die Ausbildung der Zivildiener während des Grundlehrganges sollte motivierend } \\
\text { wirken. }\end{array}$ & 0,575 & 0,655 \\
\hline V5 & $\begin{array}{l}\text { Der Zivildiener sollte während der Grundausbildung auf mögliche Gefahren und } \\
\text { Ängste vorbereitet werden. }\end{array}$ & 0,508 & 0,481 \\
\hline V6 & Die Grundausbildung der Zivildiener sollte einen hohen Praxisbezug aufweisen. & 0,577 & 0,723 \\
\hline V7 & $\begin{array}{l}\text { Das Leitbild des ÖRK sollte jedem Zivildiener auch während der Weiterbildung in der } \\
\text { Bezirksstellen vermittelt werden. }\end{array}$ & 0,510 & 0,491 \\
\hline \multicolumn{4}{|c|}{ Dimension Organisation } \\
\hline V8 & Das Rote Kreuz sollte eine ordentliche Organisationsstruktur besitzen. & 0,452 & 0,686 \\
\hline V9 & $\begin{array}{l}\text { Patienten/-innen sollten den Mitarbeitern/-innen des Roten Kreuzes vertrauen } \\
\text { können. }\end{array}$ & 0,752 & 0,517 \\
\hline V10 & $\begin{array}{l}\text { Die Mitarbeiter/-innen des Roten Kreuzes sollten angemessene Unterstützung vom } \\
\text { Rot-Kreuz - Management erhalten, um ihre Tätigkeiten gut ausführen zu können. }\end{array}$ & 0,499 & 0,598 \\
\hline V11 & $\begin{array}{l}\text { Auf der menschlichen Seite sollte ein gutes Arbeitsklima zwischen den } \\
\text { hauptamtlichen Mitarbeitern/-innen, den Freiwilligen Helfern und den Zivildienern } \\
\text { herrschen. }\end{array}$ & 0,478 & 0,522 \\
\hline \multicolumn{4}{|c|}{ Leistungsumfeld } \\
\hline V12 & Die Räumlichkeiten des ÖRK sollten ansprechend gestaltet sein. & 0,483 & 0,520 \\
\hline V13 & $\begin{array}{l}\text { Die Bezirksstelle sollte den Zivildienern saubere Räumlichkeiten zur Verfügung } \\
\text { stellen. }\end{array}$ & 0,558 & 0,491 \\
\hline V14 & $\begin{array}{l}\text { Die Weiterbildung der Zivildiener in der Bezirksstelle sollte auf die wesentlichen } \\
\text { Inhalte beschränkt sein. }\end{array}$ & 0,532 & 0,690 \\
\hline V15 & $\begin{array}{l}\text { Der Tätigkeitsbereich des Zivildieners sollte interessant und abwechslungsreich } \\
\text { gestaltet sein. }\end{array}$ & 0,623 & 0,452 \\
\hline \multicolumn{4}{|c|}{ Kundenkontakt } \\
\hline V16 & $\begin{array}{l}\text { Man sollte vom Roten Kreuz nicht erwarten, daß es den Patienten/-innen genau } \\
\text { darüber Auskunft gibt, wann die Dienstleistung ausgeführt wird. }\end{array}$ & 0,659 & 0,613 \\
\hline V17 & $\begin{array}{l}\text { Es ist unrealistisch, als Patient/-in prompten Service von den M itarbeiter/-innen des } \\
\text { Roten Kreuzes zu erwarten. }\end{array}$ & 0,630 & 0,651 \\
\hline V18 & $\begin{array}{l}\text { Vom Roten Kreuz sollte nicht erwartet werden, daß es allen Patienten/-innen } \\
\text { individuelle Aufmerksamkeit schenkt. }\end{array}$ & 0,712 & 0,536 \\
\hline V19 & $\begin{array}{l}\text { Von den Mitarbeiter/-innen des ÖRK kann nicht erwartet werden, daß sie sich } \\
\text { persönlich um die Patienten/-innen kümmern. }\end{array}$ & 0,709 & 0,572 \\
\hline V20 & $\begin{array}{l}\text { Es ist unrealistisch, von den Mitarbeiter/-innen des Roten Kreuzes zu erwarten, daß } \\
\text { sie die Bedürfnisse ihrer Patienten/-innen kennen. }\end{array}$ & 0,639 & 0,514 \\
\hline V21 & $\begin{array}{l}\text { Es ist unrealistisch zu erwarten, daß das Rote Kreuz nur das Interesse ihrer } \\
\text { Patienten/-innen im Auge hat. }\end{array}$ & 0,659 & 0,613 \\
\hline V22 & $\begin{array}{l}\text { Man sollte vom Roten Kreuz nicht erwarten, daß es den Patienten/-innen rund um die } \\
\text { Uhr Hilfe leistet. }\end{array}$ & 0,635 & 0,533 \\
\hline
\end{tabular}

Tab. 8: Faktorladungen der explorativen Faktoranalyse zur Generierung des Servqual-Item-Pools

Wie in Tabelle 8 zu sehen ist, bestätigte sich die Dimensionenqualität des Itempools (siehe Ladung t1) auch in der bereits erwähnten Zweiterhebung (La- dung t2). Dieses Ergebnis bekräftigte die Validität der Analyse und bestätigte uns darin, die Faktor-Scores als Grundlage weiterer Analysen zu verwenden. 


\subsubsection{Prüfung der SERVQUAL- Basishypothesen}

Eine wesentliche Herausforderung des ÖRK besteht darin, möglichst viele Zivildienstleistende zum Übertritt in den Freiwilligendienst zu motivieren. Bedingt durch diese Aufgabenstellung wurde erstmals in diesem Anwendungsbereich der SERVQUAL-Ansatz einges etzt. Die Wünsche der Zivildienstleistenden gehen im vorliegenden Messmodell als Bedürfnisse und Anforderungen ein. Die Ergebnisse liefern die Basis zukünftiger Managementimplikationen, die es gilt seitens des Roten Kreuzes in möglichst umfassender Form zu berücksichtigen.

Basierend auf den bereits dargestellten organisationspsychologischen Studienergebnisse, den Erkenntnissen aus der Konsumentenforschung und der Messung der Qualität von Dienstleistungen mit Hilfe des SERVQUAL-Ansatzes in Non-Profit-Bereichen (Lam, 1997; Shemwell/Yavas, 1999; Reidenbach/ Sandiffer-Smallwood, 1990) manifestiert sich eine der beiden Grundannahme der Autoren, dass die Zufried enheit mit dem Zivildienst positiv mit der Übertrittswahrscheinlichkeit der Zivildiener korreliert. Die Zufriedenheit der Zivildienstleistenden und deren Wahrscheinlichkeit eines möglichen Übertritts in den Freiwilligendienst bilden somit die beiden Basiskonstrukte bzw. die zwei Kernhypothesen dieser Untersuchung:

H1: Die Gesamtzufriedenheit der Zivildiener korreliert positiv mit der Wahrscheinlichkeit nach Beendigung des Zivildienstes in den Freiwilligendienst überzutreten.

H2: Zwischen den SERVQUAL-ZufriedenheitsWerten und der prozentuellen Gesamtzufriedenheit besteht ein positiver Zusammenhang.

\begin{tabular}{|c|c|c|c|c|}
\cline { 3 - 5 } \multicolumn{1}{c|}{} & Zufriedenheit in \% & Servqual & Übertrittswahrscheinlichkeit \\
\hline \multirow{2}{*}{ Zufriedenheit } & Pearson Corr & 1,000 & 0,686 & 0,704 \\
& Sig. (1-seitig) & $(0,000)$ & $(0,000)$ & $(0,000)$ \\
\cline { 2 - 5 } & $\mathrm{N}$ & 82 & 82 & 82 \\
\hline
\end{tabular}

Tab. 9: Korrelation der Zufriedenheit der Zivildiener in \% mit den Variablen Servqual und Übertrittswahrscheinlichkeit

Bei Betrachtung der Korrelationen (Tabelle 8) zeigt sich nicht nur ein starker Zusammenhang zwischen dem Servqual-Konstrukt und der Freiwilligenwahrscheinlichkeit, es besteht darüber hinaus eine starke Abhängigkeit zwischen dem Servqual-Ansatz und der erhobenen prozentuellen Zufriedenheit. Die Basishypothesen $\mathrm{H} 1$ und $\mathrm{H} 2$ können somit bestätigt werden.

\subsubsection{Demografische Hypothesen}

Die Bedeutung soziodemografischer Variablen bei der Messung der Qualität "traditioneller", d.h. freiwilligen Dienstleistungen wurde in zahlreichen Studien (Mummalanei, 1995; Hall/Dornan, 1990) nachgewiesen. Im Kontext unfreiwilliger Dienstleistungen sind allerdings keine empirischen Befunde verfügbar, weshalb konsequenterweise im Rahmen der Zivildienerbefragung eine Reihe demografischer Merkmale der Befragten erhoben wurde. In diesem Ab- schnitt erfolgt exemplarisch eine induktiv-statistische Überprüfung des Einflusses der sozio-demografischen Variablen Ausbildung und bisherige Vereinstätigkeit auf die Zufriedenheit bzw. Übertrittswahrscheinlichkeit der Zivildiener.

Höhere Bildung geht mit höherer Spendenbereitschaft und positiver Einstellung gegenüber NPOOrganisationen einher (vgl. Webb/Green/Brashear, 2000). Dementsprechend wurde in dieser Studie postuliert, daß Zivildienstleistende mit höherem Ausbildungsgrad höhere Zufriedenheitswerte sowie Übertrittswahrscheinlichkeiten aufweisen.

H3: Zivildiener mit Maturaabschluss weisen eine höhere Zufriedenheit als Zivildiener ohne Matura auf.

H4: Zivildiener mit Maturaabschluss weisen eine höhere Übertrittswahrscheinlichkeit als Zivildiener ohne Matura auf. 


\begin{tabular}{|c|c|c|c|c|c|c|c|}
\cline { 2 - 8 } \multicolumn{1}{c|}{} & \multicolumn{2}{c|}{ Levene-Test } & \multicolumn{3}{c|}{ T-Test } & \multicolumn{2}{c|}{ Mittlelwerte } \\
\cline { 2 - 8 } \multicolumn{1}{c|}{} & $\mathrm{F}$ & Sig. & $\mathrm{T}$ & $\mathrm{df}$ & $\begin{array}{c}\text { Sig } \\
(1-\text { - seitig) }\end{array}$ & keine Matura & Matura \\
\hline Varianzen sind gleich & 0,189 & 0,665 & 0,986 & 80 & 0,327 & 71,83 & 68,2 \\
\hline
\end{tabular}

Tab. 10: T-Test zur Überprüfung der Hypothesen H3 $(n=81)$

\begin{tabular}{|c|c|c|c|c|c|c|c|}
\cline { 2 - 8 } \multicolumn{1}{c|}{} & \multicolumn{2}{c|}{ Levene-Test } & \multicolumn{3}{c|}{ T-Test } & \multicolumn{2}{c|}{ Mittlelwerte } \\
\cline { 2 - 8 } \multicolumn{1}{c|}{} & F & Sig. & T & df & $\begin{array}{c}\text { Sig } \\
\text { (1-seitig) }\end{array}$ & keine Matura & Matura \\
\hline $\begin{array}{c}\text { Varianzen sind nicht } \\
\text { gleich }\end{array}$ & 15,817 & 0,000 & 2,463 & 68,217 & 0,016 & 55,29 & 46,70 \\
\hline
\end{tabular}

Tab. 11: T-Test zur Überprüfung der Hypothesen $\mathrm{H} 4(\mathrm{n}=83)$

Obenstehende Tabellen 10 und 11 liefern überraschenderweise keine Ünterstützung für die postulierten Hypothesen. Ein Entscheidungsansatz für die höheren Übertrittswahrscheinlichkeiten der NichtMaturanten könnte darin bestehen, daß sich insbesondere Maturanten, die ein Universitätsstudium anstreben, von der stark formalisierten Anwendungsstruktur im Rot-Kreuz-Wesen eingeschränkt fühlen. Einzelmeinungen der qualitativen Untersuchungsphase deuten auf eine derartige Interpretation hin, wiewohl diesem Aspekt in der quantitativen Fragebogenerhebung kein besonderes Argument geschenkt wurde und insofern die Vermutung rein explorativen Charakter besitzt. Weitergehende Studien erscheinen vor dem Hintergrund eines selektiven M itarbeiter-Marketings hiermit als sinnvoll.
Bezüglich des demografischen Merkmals Vereinstätigkeit wurde untersucht, inwieweit sich Zivildiener mit sozialem Engagement von jenen ohne Vereinszugehörigkeit unterscheiden.

Entsprechend der Sichtweise von Piliavin und Charng (1990), daß höhere internalisierte Werte mit Gefühlen moralischer Verpflichtung einhergehen, wurden die folgenden Hypothesen formuliert:

H5: Die Anzahl der bisherigen Vereine der Zivildiener korreliert positiv mit den Zufriedenheitsscores.

H6: Die Anzahl der bisherigen Vereine der Zivildiener korreliert positiv mit der Übertrittswahrscheinlichkeit.

\begin{tabular}{|c|c|c|c|c|c|}
\cline { 3 - 6 } \multicolumn{2}{c|}{} & $\begin{array}{c}\text { Zufriedenheit } \\
\text { in \% }\end{array}$ & Vereinstätigkeit & OLS & Involvement \\
\hline \multirow{2}{*}{$\begin{array}{c}\text { Zufriedenheit } \\
\text { in \% }\end{array}$} & Pearson Corr & 1,000 & 0,217 & 0,278 & 0,768 \\
& Sig. (1-seitig) & $(0,000)$ & $(0,025)$ & $(0,006)$ & $(0,000)$ \\
\cline { 2 - 6 } & $\mathrm{N}$ & 82 & 82 & 82 & 80 \\
\hline
\end{tabular}

Tab. 12: Korrelation der Zufriedenheit mit den Variablen Anzahl der Vereine, OLS und Involvement

\begin{tabular}{|c|c|c|c|c|c|}
\cline { 3 - 5 } \multicolumn{2}{c|}{} & $\begin{array}{c}\text { Übertrittswahr- } \\
\text { scheinlichkeit }\end{array}$ & Vereinstätigkeit & OLS & Involvement \\
\hline \multirow{2}{*}{$\begin{array}{c}\text { Übertrittswahr- } \\
\text { scheinlichkeit }\end{array}$} & Pearson Corr & 1,000 & 0,200 & 0,462 & 0,530 \\
& Sig. (1-seitig) & $(0,000)$ & $(0,034)$ & $(0,000)$ & $(0,000)$ \\
\cline { 2 - 6 } & $\mathrm{N}$ & 84 & 84 & 84 & 81 \\
\hline
\end{tabular}

Tab. 13: Korrelation der Übertrittswahrscheinlichkeit mit den Variablen Anzahl der Vereine, OLS und Involvement 
Die durchgeführten Korrelationsberechnungen weisen signifikante Ergebnisse bezüglich den beiden Basiskonstrukten auf. Es besteht somit ein positiver Zusammenhang zwischen der vorangegangenen Vereinstätigkeit von Zivildienern und ihren Zufriedenheitswerten bzw. zwischen Vereinstätigkeit und der Wahrscheinlichkeit des Übertritts in den Freiwilligendienst.

\subsubsection{Psychografische Hypo- thesen}

Personen, die selbständig Informationen suchen, häufig Empfehlungen abgeben und somit die Meinungen von anderen beeinflussen, werden als Meinungsführer oder "Opinion-Leader" bezeichnet. Diese Merkmale können entweder auf soziale Positionen, die eine überdurchschnittliche Kontaktchance bedingen oder auf Persönlichkeitsmerkmale zurückgeführt werden. Opinion-Leader sind daher einerseits durch allgemeine Eigenschaften ihrer Persönlichkeit, andererseits durch produkt- oder bereichsspezifische Merkmale gekennzeichnet (vgl. Kroeber-Riel, 1996).

Die Marketing-Bedeutung in der traditionellen $\mathrm{Be}$ triebswirtschaft des Meinungsführerkonzeptes liegt in der verhaltensrelevanten Kompetenz des Opinion-Leaders. Als instrumentelle Konsequenzen können daraus beispielsweise Strategien abgeleitet werden, die Meinungsführereigenschaften als relevante Zielgruppen- oder Segmentmerkmale ansehen und diese in der Folge bei ihrer Zielgruppenansprache auch entsprechende berücksichtigen (vgl. Engel/Blackwell/Miniard, 1995, S. 725 ff.). Das Kriterium der selektiven "aktiven Marktbearbeitung“ macht diesen Ansatz auch für die Mitarbeiterakquisition von Freiwilligen im NPO-Bereich interessant. Aus diesem Grund wurde das Konstrukt OpinionLeader-Ship (OLS) in die Untersuchung miteinbezogen und im Kontext bisheriger Untersuchungsergebnisse (Childers, 1986; Goldsmith/Desborde, 1991; Riecken/Yavas, 1983) in folgende Beziehungen zur Zivildienerzufriedenheit und Übertrittswahrscheinlichkeit gesetzt:

H7: Zivildiener mit hohen OLS Werten sind zufriedener.

H8: Zivildiener mit großer Neigung zum OLS weisenhöhere Prozentsätze in der Freiwilligenwahrscheinlichkeit auf.
Die durchgeführten Korrelationsberechnungen (siehe Tabelle 12 und 13) bestätigen die postulierten Annahmen. Es zeigt sich ein positiver Zusammenhang zwischen dem psychografischem Merkmal OpinionLeader-Ship und den Zufriedenheitswerten bzw. zwischen OLS und den Übertrittswahrscheinlichkeiten. Der Korrelationskoeffizient beträgt im ersten Fall 0,278 , im zweiten sogar 0,462 bei einer Vertrauenswahrscheinlichkeit von jeweils $99 \%$. Die Hypothesen $\mathrm{H} 7$ und $\mathrm{H} 8$ können demzufolge bestätigt werden.

Die Bedeutung des psychografischen Konstruktes Involvement als Aktivierungsgrad bzw. Motivstärke zur objektgerichteten Informationssuche, -aufnahme, -verarbeitung und -speicherung (Trommsdorff, 1993) im Kontext von Mitarbeiterführung und Managementmethoden ist unumstritten und durch zahlreiche Studien (Pickard, 1998; Weiss, 1998) belegt.

Der gegenständliche Anwendungshintergrund der Unfreiwilligkeit lässt es jedoch zweckmäßig erscheinen, das Konstrukt Involvement (vgl. McQuarrie/ Munson, 1986) im Rahmen dieser Studie erneut auf seinen Wirkungsbereich zu untersuchen.

Nachdem in bisherigen Studien (Little, 1998; McLean, 2000) ein direkter Zusammenhang zwischen Mitarbeiterinvolvement und Zufriedenheit festgestellt wurde, gehen die Autoren ebenfalls von einer positiven Korrelation zwischen dem Konstrukt Involvement und der Zufriedenheit bzw. der Übertrittswahrscheinlichkeit aus:

H9: Zivildiener mit hohem Involvement zeigen hohe Zufriedenheitswerte.

H10: Zivildiener mit hohen Involvementwerten weisen hohe Prozentsätze in der Freiwilligenwahrscheinlichkeit auf.

Tabelle 12 und 13 spiegeln die hohe Bedeutung dieses Ansatzes für den vorliegenden Anwendungsfall wider. Es konnte ein starker positiver Zusammenhang zwischen dem psychografischen Konstrukt Involvement und den beiden Basiskonstrukten der Untersuchung, Zufriedenheit (Korrelationskoeffizient $+0,768$ ) und Übertrittswahrscheinlichkeit (Korrelationskoeffizient $+0,530$ ) nachgewiesen werden. Diese beiden hochsignifikanten Ergebnisse (Vertrauenswahrscheinlichkeit $99 \%$ ) führen zur Annahme der Hypothesen $\mathrm{H} 9$ und $\mathrm{H} 10$. 


\section{Resumé - Implikationen - Forschungsausblick}

Der vorliegende Beitrag beschäftigt sich mit der Messung der internen Dienstleistungsqualität bei der Ableistung des unfreiwilligen Zivildienstes beim Österreichischen Roten Kreuz. Die Thematik ist sowohl bezüglich der Managementpraxis, als auch hinsichtlich des spezifischen Anwendungsbereiches für die wissenschaftliche Forschung von Interesse.

In methodisch-/wissenschaftlicher Hinsicht stellt dieser Beitrag eine neuartige Anwendung des SERVQUAL-Ansatzes dar. Dieses von Parasuraman, Zeithaml und Berry (1985) entwickelte Instrumentarium zur Operationalisierung von Dienstleistungsqualität wurde schon in vielen Folgestudien auf Leistungsbereiche wie etwa Krankenhäuser, Banken, Behörden etc. hin angepasst und fand mehrfach Verwendung. Der mit dem vorliegenden Beitrag hergestellte Anwendungsbezug auf "unfreiwillige“ Dienstleistungen und die Messung der „internen“ Dienstleistungsqualität blieb allerdings bislang ohne empirische Basis. Insofern vermittelt der gegenständliche Beitrag über die Dienstleistungsmessung beim Zivildienst einen wertvollen Replikations-, Validierungs- und Erweiterungsschritt für SERVQUAL.

In praktischer Hinsicht liefert das Thema insofern einen Beitrag, als die laufende Akquisition freiwilliger Mitarbeiter für die Abdeckung der gegenwärtigen und zukünftigen Leistungsangebote des ÖRK eine Grundvoraussetzung darstellt. Die Anwerbung erfolgt dabei vorwiegend über die derzeit tätigen ehrenamtlichen Mitarbeiter, über das J ugendrotkreuz, Medizinstudenten bzw. Personen, die medizinische Ausbildungen durchlaufen.

Zweifellos stellen vor allem die quasi unfreiwillig zur Mitarbeit verpflichteten Zivildiener ein großes Reservoir an potentiellen freiwilligen Mitarbeitern dar. Durch das System des Zivildienstes leisten pro J ahr mehr als 2000 Personen über eine Zeitspanne von 12 Monaten ihren Dienst beim ÖRK ab. Auf diese Weise sind Zivildiener in ein intensives Kontaktverhältnis mit dem ÖRK eingebunden. Unfreiwillig ist die Interaktion zwischen dem ÖRK und den Zivildienern deshalb, weil sie nur durch die gesetzliche Vermittlung zustande kommt. Ein Restanteil an Freiwilligkeit existiert aufgrund der Wahlmöglichkeit der Zivildiener, allenfalls andere Institutionen zur Ableistung des gesetzlich verpflichtenden Wehrersatzdienstes zu selektieren. Die systematische Analyse der Ausbildungs- und Führungsstrukturen zwischen dem ÖRK und den Zivildienern sowie der Sichtweise/Wahrnehmung der Zivildiener gegenüber dem „Dienstleister" ÖRK liefert demnach wichtige Ansatzpunkte für eine möglichst friktionsfreie und zielorientierte Beziehung, welche die Chancen auf Rekrutierung der Zivildiener für spätere freiwillige Mitarbeit beim ÖRK erhöhen kann.

Die Empirie zeigt, dass die wichtigste Informationsquelle vor Antritt der Zivildienertätigkeit Gespräche mit Freunden, Bekannten und Verwandten bzw. Mitarbeitern des ÖRK darstellen. Dies ist in Übereinstimmung mit Schildorfer (2000) und dokumentiert die zentrale Bedeutung von konstruktiver Öffentlichkeitsarbeit für das ÖRK.

Als Motive für die Wahl des Zivildienstes beim Roten Kreuz wurden sowohl persönliche als auch altruistische Motive angegeben (Abneigung gegen Bundesheer, interessante, sinnvolle Tätigkeit, Interesse und Spaß am Sozialdienst und Gewissensgründe). Hier zeigen sich kommunikationspolitische Möglichkeiten. Durch konkrete Bezugnahme auf die Sinnhaltigkeit der Rot-Kreuz Tätigkeit, der sozialen Verantwortlichkeit und des angenehmen Arbeitsklimas können die Kontakte mit dem ÖRK langfristig positiv beeinflusst werden.

Zivildiener tragen eine große Verantwortung. "Schuld zu sein, dass jemand stirbt" bedeutet für die Mehrheit der Befragten eine große Angst und psychische Belastung. Dieses Ergebnis signalisiert die Wichtigkeit von Schulungen sowohl in fachlicher als auch persönlicher Hinsicht. Bei der Ausarbeitung eines geeigneten Schulungsprogrammes sollte aber vor allem auch daran gedacht werden, wie ein allfälliges Schulungsprogramm für einen späteren Übertritt als freiwilliger Mitarbeiter gestaltet werden könnte. Für eine mögliche spätere Tätigkeit sollte die erlernten Kenntnisse und Fähigkeiten der ehemaligen Zivildiener unbedingt berücksichtigt werden und deren Einsatz entsprechend ihrem erworbenen Kompetenzen erfolgen. Eine Wiederholung der bereits schon im Rahmen der Ausübung der Zivildiensttätigkeit erlernten Inhalte sollte nur in den Bereichen, in den eine Auffrischung oder Vermittlung neuester Erkenntnisse relevant erscheint, erfolgen. J eder neue Zivildiener sollte während seiner gesamten 12-monatigen Tätigkeit über die unterschiedlichen Möglichkeiten der Gestaltung der Berufslaufbahn vom Zivildiener zum freiwilligen Sanitäter umfassend informiert und im Falle eines Interesses intensiv unterstützt und motiviert werden. 
Die zu zwei unterschiedlichen Zeitpunkten erhobenen Daten bestätigten eindeutig den postulierten Zusammenhang zwischen der Zufriedenheit der Zivildiener und deren Übertrittswahrscheinlichkeit. Hinsichtlich der Einflussfaktoren bzw. Erklärungsgründe der Zufriedenheit konnten signifikante Zusammenhänge zwischen den Zufriedenheitsscores und der Vereinszugehörigkeit, dem Involvement und hohen Meinungsführerwerten ermittelt werden. Es zeigte sich insgesamt eine relativ hohe Gesamtzufriedenheit, wobei anzumerken ist, dass durch die Tätigkeit als Zivildiener beim ÖRK die Einschätzung sich noch weiter verbesserte bzw. die Übertrittswahrscheinlichkeiten nach fünf M onaten Tätigkeit signifikant anstiegen.

Eine Weiterführung des empirischen Forschungsansatzes wäre zunächst durch Ausdehnung des geographischen Erhebungskontextes auf internationales Niveau gewinnbringend. Weiters wäre es sicherlich interessant in weiterführenden Studien mit einer dritten Erhebungswelle, z.B. nach Beendigung der gesamten 12-monatigen Zivildienstzeit, zu arbeiten. Der Erhebungszeitraum von 5 Monaten reicht möglicherweise nicht aus, um stabile Einstellungsveränderungen oder grundsätzliche Sichtweisen gegenüber dem Roten Kreuz aufzudecken. Insofern wäre interessant, welche Einstellungskonfiguration und Sichtweise unfreiwillig Dienstleistende Zivildiener nach der Gesamtdauer des Zivildienstes von 12 Monaten besitzen. Schließlich wäre es auch denkbar, die Zivildienertätigkeit in „ähnlich ausgerichteten Organisationen" zu analysieren, um herauszufinden, welche Einflussfaktoren auf die Zufriedenheit unmittelbar mit der jew eiligen Organisation verbunden sind bzw. welche sich durch die "Institution“ Zivildienst erklären lassen.

\section{Literatur}

Babakus, E./Boller, G. (1992): An Empirical Assessment of the SERVQUAL Scale, in: J ournal of Business Research, Vol. 24, Iss. 3, S. 253-268.

Carman, J. M. (1990): Consumer Perception of Service Quality, An Assessment of the SERVQUAL Dimensions, in: J ournal of Retailing, Vol. 66, S. 3357.

Childers, T. (1986): Assessment of the Psychometric Properties of an Opinion Leadership Scale, in: J ournal of Marketing Research, Vol. 23, S. 184-188.
Churchill, G. (1979): A Paradigm for Developing Better Measures of Marketing Constructs, in: J ournal of Business Research, Vol. 16, S. 64-73.

Donnelly, M/Wisniewski, M./Dalrymple, J./Curry, A. (1995): Measuring service quality in local government: The Servqual approach, in: The International J ournal of Public Sector Management, Vol. 8, Iss. 7, S. 15-20.

Engel, J ./Blackwell, R./M iniard, P. (1995): Consumer Behaviour, The Dryden Press, Orlando.

Goldsmith, R./Desborde, R. (1991): A Validity Study of a Measure of Opinion Leadership, in: J ournal of Business Research, Vol. 22, S. 11-19.

Hackman, J/Oldham, G. (1980): Work Redesign, Reading/Mass.

Hair, J . (1998): Multivariate Data Analysis, PrenticeHall International, Upper Saddle River.

Hall, J. A./Dornan, M. C. (1990), Patient Sociodemographic Characteristics as Predictors of Satisfaction with Medical Care: A Meta-Analysis, in: Social Science and Medicine, Vol. 30, S. 811-818.

Hentschel, B. (1990a): Die Messung wahrgenommener Dienstleistungsqualität mit SERVQUAL. Eine kritische Auseinandersetzung, in: Marketing ZFP, 12. J g., Heft 4, S. 230-240.

Hentschel, B. (1990b): Die Messung wahrgenommener Dienstleistungsqualität mit SERVQUAL - eine kritische Auseinandersetzung, Diskussionsbeiträge der Wirtschaftswissenschaftlichen Fakultät Ingolstadt, Nr. 3, Ingolstadt.

Höflacher, S. (1999): Wird ehrenamtliche Tätigkeit im Nonprofit Sektor durch zunehmende Professionalisierung verdrängt?, in: Witt., D. (Hrsg.) (1999): Ehrenamt und Modernisierungsdruck in Nonprofit-Organisationen, Wiesbaden, S. 51-63.

Kroeber-Riel, W. (1996): Konsumentenverhalten, Vahlen Verlag, München.

Lam, S. (1997): SERVQUAL: A tool for measuring patients opinions of hospital service quality in Hong Kong, in: Total Quality Management, Vol. 8, Iss. 4, S. 145-152. 
Lam, S./Woo, K. (1998): Measuring service quality: A test-retest reliability investigation of SERVQUAL, in: Market Research Society, Vol. 39, Iss. 2, S. 381386.

Lee, T. W./M owday, R. T. (1987): Voluntarily leaving an organization. An empirical investigation of Steers and Mowday's model of turnover, in: Academy of Management J ournal, Vol. 30, S. 721-743.

Little, G. (1998): People and Profits: Improving Involvement, in: New Zealand Manufucturer, S. 8-9.

Mayrhofer, W. (1996): Motivation und Arbeitsverhalten, in Kasper, H./Mayrhofer, W. (Hrsg.) (1996): Personalmanagement, Führung, Organisation, Wien, S. 225-255.

McLean, J./Andrew, T. (2000): Commitment, satisfaction, stress and control among social services managers andsocial workers in the UK, in Administration in Social Work, Vol. 23, S. 93.

McQuarrie, E./Munson, J . (1991): A Revised Product Involvement Inventory: Improved Usability and Validity, in: Advances in Consumer Research, Vol. 19, S. 108-115.

Mummalanei, V./Gopalakrishna, P. (1995): Mediators vs. Moderators of Patient Satisfaction, in: J ournal of Health Care, Vol. 15, No. 4, Winter 1995, S. 1623.

Llosa, S./Chandon, J ./Orsingher, C. (1998): An empirical study of SERVQUAL's dimensionality, in: The Service Industries J ournal, Vol. 18, Iss. 2, S. 195208.

Orwig, R./Pearson, J./Cochran, D. (1997): An empirical investigation into the validity of SERVQUAL into the public sector, in: Public Administration Quarterly, Vo. 27, Iss. 1, S. 54-58.

Parasuraman, A./Zeithaml, V. A./Berry, L. L. (1985): A Conceptual Model of Service Quality and ist Implications for Future Research, in: J ournal of Marketing, Vol. 49, 1985,S. 41-50.

Parasuraman, A./Zeithaml, V. A./Berry, L. L. (1988): SERVQUAL. A Multi-Item Scale for Measuring Consumer Perceptions of Service Quality, in: J ournal of Retailing, Vol. 64, No. 1, S. 12-40.
Parasuraman, A./Zeithaml, V. A./Berry, L. L. (1988): Refinement and Reassessment of the SERVQUAL Scale, in: J ournal of Retailing, Vol. 10, Iss. 4, S. 420450.

Pearce, J . (1993): Volunteers; The organizational behavior of unpaid workers, London/New York.

Pickard, J . (1998): New Management methods prove boon to productivity', in: People Management, London, October 1998.

Piliavin, J ./Charng, H. (1990): Altruism: A Review of Recent Theory and Research, in: Annual Review of Sociology, Vol. 16, S. 27-65.

Reidenbach E./Sandiffer-S mallood B. (1990): Exploring Perceptions of Hospital Operations by a Modified SERVQUAL Approach, in: J ournal of Healtch Care Marketing, Vol. 10, No. 4, S. 47-55.

Riecken, G./Yavas, U. (1983): Internal Cnsistency of King and Summers' OpinionLeadership Scale: Further Evidence, in: J ournal of Marketing Research, Vol. 20, S. 325-326.

Roßbach-Emden, B./Gaalken, K./Pauli, C. (1995): Messung der Dienstleistungsqualität der Personalarbeit als Grundlage für ein Qualitätsmanagement - am Beispiel ABB, in: Internes Marketing: Integration der Kunden- und Mitarbeiterorientierung, Wiesbaden, 1995.

Schildorfer, C. (2000): Motivation und Motivierung ehrenamtlicher Mitarbeiter in Nonprofit Organisationen am Beispiel des Österreichischen Roten Kreuzes, Wiener Neustadt, unveröff. Diplomarbeit, 2000.

Schwaab, M.-O. (1993): Erwartungen an einen Arbeitgeber, in: Moser, K./Stehle, W./Schuler, H. (Hrsg.): Personalmarketing. Beiträge zur Organisationspsychologie (Bd. 9), Stuttgart, S. 1929.

Shemwell, D./Yavas, U. (1999): Measuring service quality in hospitals: Scale development and managerial applications, in: J ournal of Marketing Theory and Practice, Vol. 7, Iss. 1, S. 15.

Strümpel, C./Pleschberger, S./Riedel, B. (1999): Freiwilliges Engagement in Niederösterreich,. Status quo und internationale Beispiele der Förderung, Wien und St. Pölten. 
Steers, R. M./Mowday, R. T. (1981): Employee, turnover and post-decision accommodation processes, in: Cummings, L. L./Staw, B. M. (Hrsg.): Research in Organizational Behavior, Vol. 3, Greenwich.

Teas, R. K. (1993): Consumer Expectations and the Measurement of Preceived Service Quality, in: J ournal of Professional Services Marketing, Vol. 8, No. 2, S. 33-53.

Trommsdorff, V. (1993): Konsumentenverhalten, Stuttgart.

V. Eckardstein, D. (1997): Personalmanagement in NPOs, in: Badelt, C. (Hrsg.)(1997) Handbuch der
Nonprofit Organisation, Stuttgart, S. 257-276.

v. Rosenstiel, L. (2000): Grundlagen der Organisationspsychologie, Schäffer-Poeschel Verlag, Stuttgart.

Webb, D./Green, C./Brashear, T. (2000):Development and Validation of Scales to Measure Attitudes Influencing Monetary Donations to Charitable Organisations, in: J ournal of Academic Marketing Science, Vol. 28, No. 2, S. 299-309.

Weiss, W. (1998): Employee involvement, commitment and cooperation: Keys to successful supervision, in: Supervision, Vol. 59,S. 12-15. 